TRANSACTIONS OF THE

AMERICAN MATHEMATICAL SOCIETY

Volume 350, Number 6, June 1998, Pages 2297-2310

S 0002-9947(98)02218-1

\title{
SINGULARITY OF SELF-SIMILAR MEASURES WITH RESPECT TO HAUSDORFF MEASURES
}

\author{
MANUEL MORÁN AND JOSÉ-MANUEL REY
}

\begin{abstract}
Besicovitch (1934) and Eggleston (1949) analyzed subsets of points of the unit interval with given frequencies in the figures of their base- $p$ expansions. We extend this analysis to self-similar sets, by replacing the frequencies of figures with the frequencies of the generating similitudes. We focus on the interplay among such sets, self-similar measures, and Hausdorff measures. We give a fine-tuned classification of the Hausdorff measures according to the singularity of the self-similar measures with respect to those measures. We show that the self-similar measures are concentrated on sets whose frequencies of similitudes obey the Law of the Iterated Logarithm.
\end{abstract}

\section{INTRODUCTION}

The research developed in this article is motivated by the following well-known example. Let $I=[0,1] \subset \mathbb{R}$, and take an integer $m>1$. Let $M=\{0,1, \ldots, m-1\}$. For $x \in I$ and $j \in M$, let $\delta_{j}(x)$ be the asymptotic frequency of the integer $j$ in the non-terminanting base- $m$ expansion of $x, x=\sum_{k=1}^{\infty} j_{k} m^{-k}$ with $j_{k} \in M$. Given a set of positive numbers $\left\{p_{0}, \ldots, p_{m-1}\right\}$ such that $\sum_{j \in M} p_{j}=1$, consider the set $\Lambda_{m}\left(p_{0}, \ldots, p_{m-1}\right) \subset I$ composed of those $x$ such that $\delta_{j}(x)=p_{j}$ for $j \in M$.

In 1934 Besicovitch proved that the Hausdorff dimension of the set $\Lambda_{2}(p, 1-p)$ is given by $-\log _{2}\left(p^{p}(1-p)^{1-p}\right)$ [3]. Eggleston generalized this formula for $m>2$. More precisely, he proved in [8] that the Hausdorff dimension of $\Lambda_{m}\left(p_{0}, \ldots, p_{m-1}\right)$ is given by

$$
\operatorname{dim} \Lambda_{m}\left(p_{0}, \ldots, p_{m-1}\right)=\frac{\sum_{j=0}^{m-1} p_{j} \log p_{j}}{-\log m}
$$

Henceforward the sets $\Lambda_{m}\left(p_{0}, \ldots, p_{m-1}\right)$ are called Besicovitch-Eggleston sets.

In 1960 Billingsley [4] considered Hausdorff measures on a probability space $(\Omega, \mathcal{A}, \mu)$ carrying a stochastic process $\left\{x_{1}, x_{2}, \ldots\right\}$ with a finite state space. He found the Hausdorff dimension of sets of points characterized by the asymptotic frequency of the transitions between the states when the process $\left\{x_{1}, x_{2}, \ldots\right\}$ is a regular Markov chain. The probability space $(\Omega, \mathcal{A}, \mu)$ can be chosen to be the unit interval in the real line with the Lebesgue measure, and then the results of Besicovitch and Eggleston turn out to be a particular case of those of Billingsley.

Received by the editors January 17, 1996.

1991 Mathematics Subject Classification. Primary 28A78, 28A80.

Key words and phrases. Self-similarity, Hausdorff measures, dimension function, Law of the Iterated Logarithm.

Research partially supported by Ente Público Puertos del Estado.

(C)1998 American Mathematical Society 
Iterated function systems with probabilities [2] can be regarded as geometrical realizations of the scheme of Billingsley. There is a natural extension of the definition of the Besicovitch-Eggleston sets in this context, as sets of points with given asymptotic frequencies of their generating contractions. In the case when the contractions are similitudes satisfying a suitable separation condition, the formula (1.1) for the dimension of the Besicovitch-Eggleston sets can be generalized in a natural way. This can be derived from results in $[5,7]$ and Theorem 2.1 in this paper (see section 2).

Self-similar measures [10] can be considered as geometric projections of product probability measures from an abstract Cantor set. In this paper we analyze the relationships among such measures, Hausdorff measures, and certain sets that, as the Besicovitch-Eggleston sets, are characterized by the asymptotic properties of their generating similitudes. In section 2 we describe the main results of the paper, and we give the proofs in section 3 .

\section{MAin Results}

Let $\Psi=\left\{\varphi_{i}: i \in M\right\}$, with $M=\{1,2, \ldots, m\}$, be a system of contractive similitudes of $\mathbb{R}^{N}$, where $N$ is a positive integer. We call $\mathcal{S}(N, M)$ the set of such systems. There exists a unique compact set $E$ such that $E=S \Psi(E):=\bigcup_{i \in M} \varphi_{i}(E)$ [10], which is called the self-similar set generated by $\Psi$. There exists a surjective mapping $\pi$ from the product space $M^{\infty}:=M \times M \times \ldots$ onto $E$ given by

$$
\pi(\mathbf{i})=\bigcap_{k=1}^{\infty}\left(\varphi_{i_{1}} \circ \varphi_{i_{2}} \circ \cdots \circ \varphi_{i_{k}}(E)\right)
$$

for $\mathbf{i}=\left(i_{1} i_{2} \ldots\right) \in M^{\infty}$. Let $s$ be the unique real number such that $\sum_{i \in M} r_{i}^{s}=1$, where $r_{i}$ is the contraction ratio of $\varphi_{i}$ for $i \in M$. We assume that the standard open set condition (OSC) holds for the system $\Psi$ (see section 3 ). It is known that the Hausdorff dimension of $E$ is given by $s$ and its $s$-dimensional Hausdorff measure is positive and finite [10].

Let $\mathcal{P}^{+} \subset \mathbb{R}^{m}$ be the set of positive probability vectors in $M$, that is, $\mathbf{p} \in \mathcal{P}^{+}$ if and only if $\mathbf{p}=\left(p_{i}: i \in M\right)$ with $\mathbf{p}(i)=p_{i}>0$ for all $i$ and $\sum_{i \in M} p_{i}=1$. Given $\mathbf{p} \in \mathcal{P}^{+}$, we denote by $\nu_{\mathbf{p}}$ the probability measure on $M^{\infty}$ defined by the infinite-fold product measure $\mathbf{p} \times \mathbf{p} \times \ldots$ Let $\mu_{\mathbf{p}}$ be the projected product measure supported by $E$, that is $\mu_{\mathbf{p}}=\nu_{\mathbf{p}} \circ \pi^{-1}$. Let $\mathcal{M}^{+}=\left\{\mu_{\mathbf{p}}: \mathbf{p} \in \mathcal{P}^{+}\right\}$be the set of such measures on $E$, also called self-similar measures [1]. We will write $\mathbf{p}_{s}$ for the choice $\mathbf{p}=\left(r_{i}^{s}: i \in M\right)$. In [7] it was proved that the Hausdorff dimension of a measure $\mu_{\mathbf{p}} \in \mathcal{M}^{+}$is given by

$$
\operatorname{dim} \mu_{\mathbf{p}}=s(\mathbf{p}):=\frac{\sum_{i \in M} p_{i} \log p_{i}}{\sum_{i \in M} p_{i} \log r_{i}}
$$

where $\operatorname{dim} \mu=\inf \{\operatorname{dim} A: \mu(A)=1, A$ a Borel set $\}$ and $\operatorname{dim}(\cdot)$ stands for the Hausdorff dimension (see section 3 for a definition). Observe that $s(\mathbf{p})=s$ for $\mathbf{p}=\mathbf{p}_{s}$.

We now formulate the main results in this paper. Let $\Theta$ be the overlapping set of $\Psi$, that is,

$$
\Theta=\bigcup_{i \neq j}\left(\varphi_{i}(E) \cap \varphi_{j}(E)\right)
$$


The following theorem will be proved in section 3 and will be used throughout the paper. An independent proof has been given by N. Patzschke [14].

Theorem 2.1 ( $\Theta$-lemma). $\mu(\Theta)=0$ for all $\mu \in \mathcal{M}^{+}$.

Theorem 2.1 solves a problem posed by C. Bandt [1] about the singularity of selfsimilar measures when $\pi$ is not injective. It is well-known that different choices of $\mathbf{p}$ yield measures $\nu_{\mathbf{p}}$ on $M^{\infty}$ which are mutually singular (see e.g. [21]). This obviously implies that the induced self-similar measures $\mu_{\mathbf{p}}$ are also mutually singular when $\pi$ is a bijection. Whether the same property holds when $\pi$ fails to be an injection is an open problem [1]. The result above shows that, under the OSC, two distinct self-similar measures are mutually singular. Furthermore, Falconer [9] and Cawley and Mauldin [5] have pointed out the convenience of a result such as Theorem 2.1 in order to extend multifractal analysis of self-similar measures performed for disjointed constructions.

Let $H^{\phi}$ denote the $\phi$-Hausdorff measure associated with a dimension function $\phi$ (see section 3 for a definition). We write $H^{t}$ for $\phi(\xi)=\xi^{t}$. We now state the main result in this paper, which concerns the geometry of self-similar measures. Observe that it cannot be decided from (2.1) whether the measure $\mu_{\mathbf{p}}$ is singular or absolutely continuous with respect to the Hausdorff measure $H^{s(\mathbf{p})}$. The following theorem can be proved, which answers this problem by means of a fine-tuned classification of Hausdorff measures.

Theorem 2.2. Let $\Psi \in \mathcal{S}(N, M)$ satisfying the $O S C$ and $\mathbf{p} \in \mathcal{P}^{+} \backslash\left\{\mathbf{p}_{s}\right\}$ be given. Let $\alpha \geq 0$, and let $\mathcal{G}=\left\{\phi_{\alpha}\right\}_{\alpha \geq 0}$ be the one-parameter family of real functions

$$
\phi_{\alpha}(\xi)=\xi^{s(\mathbf{p})} \exp \left\{\alpha\left(2 \log \xi^{c(\mathbf{p})} \log \log \log \xi^{c(\mathbf{p})}\right)^{1 / 2}\right\},
$$

where $s(\mathbf{p})$ is given in $(2.1)$, and

$$
c(\mathbf{p})=\left(\sum_{i \in M} p_{i} \log r_{i}\right)^{-1}<0 .
$$

Let

$$
d(\mathbf{p})=\left(\sum_{i \in M}\left(\log p_{i}-s(\mathbf{p}) \log r_{i}\right)^{2} p_{i}\right)^{1 / 2} .
$$

Then, for the self-similar measure $\mu_{\mathbf{p}}$ induced by $\mathbf{p}$, the following assertions hold:

(i) $\mu_{\mathbf{p}}$ is singular with respect to (w.r.t.) $H^{\phi_{\alpha}}$ if $0 \leq \alpha<d(\mathbf{p})$;

(ii) $\mu_{\mathbf{p}}$ is absolutely continuous w.r.t. $H^{\phi_{\alpha}}$ if $\alpha>\bar{d}(\mathbf{p})$;

(iii) $\mu_{\mathbf{p}}$ has an integral representation w.r.t. a Hausdorff measure $H^{t}$ if and only if $\mathbf{p}=\mathbf{p}_{s}$, and thus $t=s$. In case $\mathbf{p}=\mathbf{p}_{s}$, then $\mu_{\mathbf{p}_{s}}=\left(H^{s}(E)\right)^{-1} H^{s}\left\lfloor_{E}\right.$, where $H^{s}\left\lfloor_{E}\right.$ stands for the restriction of the s-dimensional Hausdorff measure to the set E.

Remark 2.3. A result akin to Theorem 2.2 was obtained by Przytycki, Urbański and Zdunik [15] for Gibbs and harmonic measures on certain repellers in the complex plane. The dimension function they found is contained in the family (2.3). Also, Smorodinsky [19] showed, for the binary Besicovitch-Eggleston sets, that self-similar measures cannot be proportional to a Hausdorff measure $H^{\phi}$ if the function $\phi$ is concave near the origin.

Since $H^{s(\mathbf{p})} \leq H^{\phi_{\alpha}}$ for all $\alpha>0$, Theorem 2.2 implies that, for $\mathbf{p} \neq \mathbf{p}_{s}$, the selfsimilar measure $\mu_{\mathbf{p}}$ is singular with respect to the Hausdorff measure $H^{s(\mathbf{p})}$. On the 
other hand, Theorem 2.2 allows us to discern which of two given measures with the same dimension is concentrated on a "smaller" set (in the sense of the $\phi$-Hausdorff measure). Notice that $s(\mathbf{p})=s(\mathbf{q})$ for $\mathbf{p} \neq \mathbf{q}$ is a plausible case. In such a case, the discriminating parameter is the standard deviation $d(\mathbf{p})$ of a random variable which depends on the pair $\left(\Psi, \mu_{\mathbf{p}}\right)$. More precisely, if $s(\mathbf{p})=s(\mathbf{q})$ for $\mathbf{p} \neq \mathbf{q}$ but $d(\mathbf{p})>d(\mathbf{q})$, then $H^{\phi_{\alpha}}(B)=+\infty$ for any set $B$ of positive $\mu_{\mathbf{q}}$-measure and for all $d(\mathbf{p})>\alpha>d(\mathbf{q})$, whereas $H^{\phi_{\alpha}}(D)=0$ for a set $D$ of full $\mu_{\mathbf{p}}$-measure. Thus, if $d(\mathbf{p})>d(\mathbf{q})$, the measure $\mu_{\mathbf{p}}$ is concentrated on a smaller set (from a geometric point of view) than $\mu_{\mathbf{q}}$ is. In case $s(\mathbf{p})=s(\mathbf{q})$ and $d(\mathbf{p})=d(\mathbf{q})$ further work is needed to solve the problem.

We next turn our attention to some natural sets that concentrate the measure $\mu_{\mathbf{p}}$, thus providing an alternative approach to self-similar geometry, different from that supplied by self-similar measures.

For $j \in M$ and $\mathbf{i}=\left(i_{1}, i_{2}, \ldots\right) \in M^{\infty}$, let $\delta_{j}(\mathbf{i})$ be the asymptotic frequency of the symbol $j$ in the sequence $\mathbf{i}$, i.e.

$$
\delta_{j}(\mathbf{i})=\lim _{n \rightarrow \infty} \frac{1}{n} \operatorname{card}\left\{k: i_{k}=j, 1 \leq k \leq n\right\} .
$$

We define the normal Besicovitch set of $\Psi \in \mathcal{S}(N, M)$ associated with the probability vector $\mathbf{p}=\left(p_{j}\right)_{j \in M}$ to be the subset of $E$ given by

$$
B_{\mathbf{p}}=\left\{\pi(\mathbf{i}): \mathbf{i} \in M^{\infty}, \delta_{j}(\mathbf{i})=p_{j} \text { for all } j \in M\right\} .
$$

The Hausdorff dimension of the sets $B_{\mathbf{p}}$ is shown to be $s(\mathbf{p})$. This is in essence a consequence of the law of large numbers, formula (2.1), and the $\Theta$-lemma. In particular, since $\mu_{\mathbf{p}}\left(B_{\mathbf{p}}\right)=1$ because of the Strong Law of Large Numbers, (2.1) provides a lower bound for the dimension of $B_{\mathbf{p}}$. An argument involving suitable coverings of the set $B_{\mathbf{p}}$ could be used to prove that $\operatorname{dim} B_{\mathbf{p}}=s(\mathbf{p})$, provided that the overlapping set $\Theta$ has zero $\mu_{\mathbf{p}}$-measure (which is the content of Theorem 2.1). This dimension result essentially follows from a theorem in multifractal analysis by Cawley and Mauldin (see [5, Theorem 2.1]).

For $\mathbf{j} \in M^{k}:=M \times \stackrel{(k)}{\cdots} \times M$, let $p_{\mathbf{j}}=p_{j_{1}} p_{j_{2}} \cdots p_{j_{k}}$, and let $\delta_{\mathbf{j}}(\mathbf{i})$ denote the asymptotic frequency of appearance of the finite sequence $\mathbf{j}$ in the infinite code $\mathbf{i} \in M^{\infty}$, i.e.

$$
\delta_{\mathbf{j}}(\mathbf{i})=\lim _{n \rightarrow \infty} \frac{1}{n} \operatorname{card}\left\{q: i_{q}=j_{1}, i_{q+1}=j_{2}, \ldots, i_{q+k-1}=j_{k}, 1 \leq q \leq n-k+1\right\} .
$$

We also define the supernormal Besicovitch set $B_{\mathbf{p}}^{(\infty)}$ of $\Psi \in \mathcal{S}(N, M)$ associated with $\mathbf{p}$ by

$$
B_{\mathbf{p}}^{(\infty)}=\bigcap_{k=1}^{\infty}\left\{\pi(\mathbf{i}): \mathbf{i} \in M^{\infty}, \delta_{\mathbf{j}}(\mathbf{i})=p_{\mathbf{j}}, \text { for all } \mathbf{j} \in M^{k}\right\}
$$

Birkhoff's ergodic theorem implies that $\mu_{\mathbf{p}}\left(B_{\mathbf{p}}^{(\infty)}\right)=1$, and thus $\operatorname{dim} B_{\mathbf{p}}^{(\infty)} \geq s(\mathbf{p})$ from (2.1). Since $B_{\mathbf{p}}^{(\infty)} \subset B_{\mathbf{p}}$, then $\operatorname{dim} B_{\mathbf{p}}^{(\infty)}=s(\mathbf{p})$.

Let $\tau$ be the shift mapping on $M^{\infty}$, i.e.

$$
\tau\left(i_{1}, i_{2}, i_{3}, \ldots\right)=\left(i_{2}, i_{3}, \ldots\right) .
$$


Given a random variable $Z: M \rightarrow \mathbb{R}$ and a sequence $\mathbf{i}=\left(i_{1}, i_{2}, \ldots\right) \in M^{\infty}$, let

$$
S_{n}^{Z}(\mathbf{i})=\sum_{j=1}^{n} Z \circ \operatorname{pr}_{1} \circ \tau^{j-1}(\mathbf{i})
$$

where $\mathrm{pr}_{1}$ is the projection mapping $M^{\infty} \rightarrow M$ onto the first coordinate:

$$
\operatorname{pr}_{1}\left(i_{1}, i_{2}, \ldots\right)=i_{1} \text {. }
$$

Let $X$ be the random variable $M \rightarrow \mathbb{R}$ defined by

$$
X(i)=\log p_{i}-s(\mathbf{p}) \log r_{i}, i \in M .
$$

Let $\mathcal{E}[\cdot]$ denote the expectation, with respect to the probability $\mathbf{p}$, of a random variable defined in $M$. Observe that $\mathcal{E}[X]=0$ and $\mathcal{E}\left[X^{2}\right]=d(\mathbf{p})^{2}$, where $d(\mathbf{p})$ is defined in (2.5). Consider the set $L_{\mathbf{p}}$ defined by

$$
\begin{aligned}
L_{\mathbf{p}}=\left\{\pi(\mathbf{i}) \in B_{\mathbf{p}}^{(\infty)}: \mathbf{i} \in M^{\infty},\right. & \limsup _{n \rightarrow \infty}\left\{(2 n \log \log n)^{-1 / 2} S_{n}^{X}(\mathbf{i})\right\}=d(\mathbf{p}), \\
& \left.\liminf _{n \rightarrow \infty}\left\{(2 n \log \log n)^{-1 / 2} S_{n}^{X}(\mathbf{i})\right\}=-d(\mathbf{p})\right\} .
\end{aligned}
$$

The set $L_{\mathbf{p}}$ carries the whole $\mu_{\mathbf{p}}$-measure, because of the Law of the Iterated Logarithm. This permits us to show that $\operatorname{dim} L_{\mathbf{p}}=s(\mathbf{p})$, since $L_{\mathbf{p}} \subset B_{\mathbf{p}}$. Furthermore, the set $L_{\mathbf{p}}$ characterizes a null $H^{s(\mathbf{p})}$-measure set concentrating $\mu_{\mathbf{p}}$, which is guaranteed to exist by virtue of Theorem 2.2 part (i).

We collect the above facts, as well as others concerning the Hausdorff measure, in

Theorem 2.4. Let $\mathbf{p} \in \mathcal{P}^{+}$. Let $B_{\mathbf{p}}, B_{\mathbf{p}}^{(\infty)}$, and $L_{\mathbf{p}}$ be the sets defined in (2.6), (2.8), and (2.13) respectively. Then

(i) $\operatorname{dim} B_{\mathbf{p}}=\operatorname{dim} B_{\mathbf{p}}^{(\infty)}=s(\mathbf{p})$.

(ii) $0<H^{s}\left(B_{\mathbf{p}_{s}}\right)=H^{s}\left(B_{\mathbf{p}_{s}}^{(\infty)}\right)=H^{s}\left(L_{\mathbf{p}_{s}}\right)=H^{s}(E)<+\infty$.

(iii) For any $\mathbf{p} \neq \mathbf{p}_{s}$, the $H^{s(\mathbf{p})}$-measure of the set $B_{\mathbf{p}}^{(\infty)}$ is either zero or infinity. If $\operatorname{dim}\left(B_{\mathbf{p}} \cap \Theta\right)<s(\mathbf{p})$, then the $H^{s(\mathbf{p})}$-measure of the set $B_{\mathbf{p}}$ is either zero or infinity.

(iv) Let $\mathbf{p} \neq \mathbf{p}_{s}$. Then $L_{\mathbf{p}}$ is a set of full $\mu_{\mathbf{p}}-$ measure, and Hausdorff dimension $s(\mathbf{p})$. Furthermore, $H^{s(\mathbf{p})}\left(L_{\mathbf{p}}\right)=0$.

Part (ii) of Theorem 2.4 provides a good reason to regard the points of $B_{\mathbf{p}_{s}}$ as the "normal" points (in the sense of Borel) of the self-similar set $E$. Moreover, the set $B_{\mathbf{p}_{s}}^{(\infty)}$ of the "super-normal" points in $E$ can be thought of as the true "core" of the set $E$. Part (iii) is a consequence of a stronger result on $S \Psi$-invariant sets (see Proposition 3.7). It follows from that result that there is no $S \Psi$-invariant $t$-set (that is, with finite and positive $H^{t}$-measure) in $E$ for $\operatorname{dim} \Theta<t<s$.

In order to see that Besicovitch-Eggleston sets are obtained as a particular case of our scheme, let $M=\{0,1, \ldots, m-1\}$, and consider the system of similitudes $\Psi=\left\{\varphi_{i}: i \in M\right\}$ on $I=[0,1]$, where $\varphi_{i}(x)=m^{-1}(x+i)$ for $i \in M$. Then $I$ is the self-similar set generated by $\Psi$, and $\Lambda_{m}\left(p_{0}, \ldots, p_{m-1}\right)$ is the Besicovitch set $B_{\mathbf{p}}$ of $\Psi$ associated with $\mathbf{p}=\left(p_{0}, \ldots, p_{m-1}\right)$ as defined in (2.6). The Hausdorff dimension of $\Lambda_{m}\left(p_{0}, \ldots, p_{m-1}\right)$ given in (1.1) is obtained from (2.1) by substituing $r_{i}=m^{-1}$ for every $i$. Therefore (2.1) generalizes formula (1.1) to self-similar sets.

The problem of finding the exact Hausdorff measure of the Besicovitch-Eggleston sets in their dimension has been asked by Mauldin on several occassions. Cooper has 
recently proved [6] that the Besicovitch-Eggleston sets actually have infinite Hausdorff measure in their dimension except for degenerate cases. A natural conjecture therefore is that self-similar Besicovitch normal sets also have infinite Hausdorff measure. Part (iii) of Theorem 2.4 reinforces this claim.

Remark 2.5. We remark here that the dimension formula in (2.1) can be interpreted in terms of the ergodic theory of dynamical systems. More particularly, we pursue an explanation of $s(\mathbf{p})$ in the spirit of [22]. See [21] for definitions.

Let $\mathbf{p} \in \mathcal{P}^{+}$and consider the dynamical system $\left(E, T, \mu_{\mathbf{p}}\right)$, where $T$ is a shift mapping on the self-similar set; that is, $T$ is defined by $\left.T\right|_{E \backslash \Theta^{*}}=\pi \circ \tau \circ \pi^{-1}, \tau$ being the Bernoulli shift on $M^{\infty}$ defined in (2.9). From the $\Theta$-lemma we learn that the measure spaces $\left(M^{\infty}, \nu_{\mathbf{p}}\right)$ and $\left(E, \mu_{\mathbf{p}}\right)$ are isomorphic (both endowed with the $\sigma$-algebra generated by the class of cylinders; see Remark 3.2). Moreover, $T$ and $\tau$ are isomorphic measure-preserving transformations. Therefore $\mu_{\mathbf{p}}$ and $\nu_{\mathbf{p}}$ have the same measure-theoretic entropy, given by $h\left(\mu_{\mathbf{p}}\right)=-\sum_{j \in M} p_{j} \log p_{j}$ (see [21]).

Raghunathan's version of Oseledec's Theorem [16] allows us to compute the whole Liapunov spectrum of the system $\left(E, T, \mu_{\mathbf{p}}\right)$. Specifically, for $x \in \varphi_{i}(E) \backslash \Theta$ define $S(x)=D_{x} \varphi_{i}^{-1}$ where $D_{x}(\cdot)$ denotes the Jacobian matrix at $x$, and let $S_{x}^{(n)}=$ $S\left(T^{n-1}(x)\right) \circ S\left(T^{n-2}(x)\right) \circ \cdots \circ S(x)$. Notice that $T(x)=\varphi_{i}^{-1}(x)$ for $x \in \varphi_{i}(E) \backslash \Theta$. The Strong Law of Large Numbers and the $\Theta$-lemma imply that, for $\mu_{\mathbf{p}}$-a.e. $x \in E$ and for all $v \in \mathbb{R}^{N}$,

$$
\lim _{n \rightarrow \infty} \frac{1}{n} \log \left\|S_{x}^{(n)} v\right\|=\lim _{n \rightarrow \infty} \frac{1}{n} \log \prod_{k=1}^{n} r_{i_{k}}^{-1}\|v\|=-\sum_{j \in M} p_{j} \log r_{j} \stackrel{\text { def }}{=} \lambda\left(\Psi, \mu_{\mathbf{p}}\right) .
$$

Oseledec's Theorem thus asserts that all the $N$ Liapunov exponents of the measurable dynamics $\left(T, \mu_{\mathbf{p}}\right)$ coincide $\mu_{\mathbf{p}}$-a.e. and are given by $\lambda\left(\Psi, \mu_{\mathbf{p}}\right)$. Taking these facts into account, the formula (2.1) for $s(\mathbf{p})$ can be written as

$$
\operatorname{dim} \mu_{\mathbf{p}}=\frac{h\left(\mu_{\mathbf{p}}\right)}{\lambda\left(\Psi, \mu_{\mathbf{p}}\right)} \text {. }
$$

Observe that the right-hand side of formula (2.14) is always defined for any $\mu_{\mathbf{p}} \in$ $\mathcal{M}^{+}$.

\section{Proofs}

We first give some basic definitions and notation.

Given $A \subset \mathbb{R}^{N}$ and $\delta>0$, a $\delta$-covering of the set $A$ is a countable collection of sets $\left\{U_{i}\right\}_{i}$ such that $\bigcup_{i} U_{i} \supset A$ with $\left|U_{i}\right| \leq \delta$, where $|\cdot|$ stands for the diameter of a set. Let $\mathcal{F}$ denote the set of dimension functions, defined by

$$
\mathcal{F}=\left\{\phi:(0, \eta) \rightarrow \mathbb{R}^{+}: \phi \text { continuous, increasing, } \lim _{\xi \rightarrow 0^{+}} \phi(\xi)=0,0<\eta<1\right\}
$$

We will use the spherical $\phi$-Hausdorff measure throughout this paper, i.e. for $\phi \in \mathcal{F}$, let

$$
H^{\phi}(A)=\sup _{\delta>0} \inf \left\{\sum_{i} \phi\left(\left|B_{i}\right|\right):\left\{B_{i}\right\}_{i} \text { is a } \delta \text {-covering of } A \text { by balls }\right\} .
$$

The expression behind the supremum is denoted by $H_{\delta}^{\phi}(A)$. For $\phi \in \mathcal{F}$ satisfying

$$
\limsup _{\xi \rightarrow 0^{+}} \frac{\phi(2 \xi)}{\phi(\xi)}=\phi^{*}<+\infty
$$


$H^{\phi}$ is comparable to the standard $\phi$-Hausdorff measure $\mathbf{H}^{\phi}$, in the sense that

$$
\left(\phi^{*}\right)^{-1} H^{\phi}(A) \leq \mathbf{H}^{\phi}(A) \leq H^{\phi}(A)
$$

for $A \subset \mathbb{R}^{N}$.

Let $H^{a}$ be the Hausdorff measure associated with the dimension function $\phi(\xi)=$ $\xi^{a}$. The Hausdorff dimension $\operatorname{dim} A$ of a subset $A \subset \mathbb{R}^{N}$ is given by the threshold value

$$
\operatorname{dim} A=\sup \left\{a: H^{a}(A)>0\right\}=\inf \left\{a: H^{a}(A)<+\infty\right\} .
$$

We call $U$ and $u$, respectively, the maximum and minimum of the set $\left\{r_{i}: i \in M\right\}$ (recall that $r_{i}$ is the contraction ratio of the similitude $\varphi_{i}$ ). We assume that $U<1$.

We say that $\Psi$ fulfills the open set condition if there exists a nonempty bounded open set $V \subset \mathbb{R}^{N}$ such that

$$
S \Psi(V) \subseteq V
$$

and

$$
\varphi_{i}(V) \cap \varphi_{j}(V)=\emptyset \text { for } i, j \in M, i \neq j .
$$

We write $F$ for the closure of $V, \bar{V}$. We may assume without loss of generality that $|V|=1$.

We write $M^{*}$ for the set of finite sequences with terms in $M$. Given a sequence $\mathbf{j}=\left(j_{1}, j_{2}, \ldots, j_{k}\right) \in M^{k}, \varphi_{\mathbf{j}}$ stands for the composite similitude $\varphi_{j_{1}} \circ \varphi_{j_{2}} \circ \cdots \circ \varphi_{j_{k}}$, and $r_{\mathbf{j}}$ stands for its contraction ratio $r_{j_{1}} r_{j_{2}} \cdots r_{j_{k}}$. The set of sequences of $M^{\infty}$ whose first $k$ figures are those of $\mathbf{j}$, that is,

$$
\left\{\left(j_{1}, j_{2}, \ldots, j_{k}, i_{k+1}, i_{k+2}, \ldots\right): i_{n} \in M, n>k\right\},
$$

is called a cylinder set. For $k \in \mathbb{N}$ and $\mathbf{i} \in M^{\infty}, \mathbf{i}(k)$ denotes the curtailed sequence $\left(i_{1} i_{2} \ldots i_{k}\right) . E_{\mathbf{j}}$ and $F_{\mathbf{j}}$ stand respectively for the image sets $\varphi_{\mathbf{j}}(E)$ and $\varphi_{\mathbf{j}}(F)$, which will also be called (geometric) cylinders.

Observe that the set

$$
\Theta^{*}=\left\{x \in E: x=\pi(\mathbf{i})=\pi(\mathbf{j}) \text { for some pair } \mathbf{i}, \mathbf{j} \in M^{\infty}, \mathbf{i} \neq \mathbf{j}\right\}
$$

can be written as the countable union $\Theta^{*}=\bigcup_{\mathbf{i} \in M^{*}} \varphi_{\mathbf{i}}(\Theta)$. In particular this implies that, for any $\mu \in \mathcal{M}^{+}$, if $\Theta$ is a $\mu$-null set, so is $\Theta^{*}$. We first give the proof of the $\Theta$-lemma.

Proof of Theorem 2.1. Since the system $\Psi$ satisfies the OSC, it is known [18] that an open set $V$ satisfying (3.4) can be chosen such that $V \cap E \neq \emptyset$ (strong OSC) holds.

Given an $x \in E$ and an $\mathbf{i} \in M^{\infty}$ such that $\pi(\mathbf{i})=x$, we define the shift $\mathbf{i}-$ orbit of $x$ as the set $\gamma_{\mathbf{i}}(x)=\left\{x, x_{1}, x_{2}, \ldots\right\}$, where $x_{m}=\varphi_{i_{m}}^{-1} \circ \cdots \circ \varphi_{i_{1}}^{-1}(x)$, for $m \geq 1$, and we define the shift orbit of $x$ as $O(x)=\bigcup\left\{\gamma_{\mathbf{i}}: \mathbf{i} \in M^{\infty}, \pi(\mathbf{i})=x\right\}$.

For $\mathbf{j} \in M^{*}$, let $\delta_{\mathbf{j}}(\mathbf{i})$ be the limit in (2.7), and consider the supernormal Besicovitch subset of the space $M^{\infty}$ :

$$
\mathcal{B}_{\mathbf{p}}^{(\infty)}=\left\{\mathbf{i} \in M^{\infty}: \delta_{\mathbf{j}}(\mathbf{i})=p_{\mathbf{j}}, \mathbf{j} \in M^{*}\right\}
$$

(recall that $p_{\mathbf{j}}=p_{j_{1}} p_{j_{2}} \cdots p_{j_{k}}$ for $\mathbf{j} \in M^{k}$ ).

Observe the following two facts.

(i) Since $\mathbf{p} \in \mathcal{P}^{+}, O(\pi(\mathbf{i}))$ is dense in $E$ for each $\mathbf{i} \in \mathcal{B}_{\mathbf{p}}^{(\infty)}$. 
(ii) $O(x) \subset \partial V$ for all $x \in \partial V \cap E$, because, otherwise, $x_{m} \in \gamma_{\mathbf{i}}(x) \cap V$ for some $m \in \mathbb{N}$ and for some $\mathbf{i} \in M^{\infty}$ would imply that $x_{m-1}=\varphi_{i_{m}}\left(x_{m}\right) \in \gamma_{\mathbf{i}}(x) \cap V$, and recursively that $x \in V$.

Assume there exists an $x \in \pi\left(\mathcal{B}_{\mathbf{p}}^{(\infty)}\right) \cap \Theta$. Since $x \in \Theta, x=\pi(\mathbf{i})=\pi(\mathbf{j})$ for $\mathbf{i}, \mathbf{j} \in M^{\infty}$ and $x \in \varphi_{i_{1}}(E) \cap \varphi_{j_{1}}(E) \subset \varphi_{i_{1}}(F) \cap \varphi_{j_{1}}(F)$ with $i_{1} \neq j_{1}$. Moreover, from the OSC, $x \in \varphi_{i_{1}}(\partial V) \cap \varphi_{j_{1}}(\partial V)$. Therefore, if $\gamma_{\mathbf{i}}(x)=\left\{x, x_{1}, x_{2}, \ldots\right\}$, then $x_{1} \in \partial V \cap E$, and $\gamma_{i_{2} i_{3} \ldots}\left(x_{1}\right) \subset \partial V$ from (ii) above. Since $\gamma_{i_{2} i_{3} \ldots}\left(x_{1}\right)$ is dense in $E$, $\partial V$ is also dense in $E$, which contradicts the strong OSC. Hence $\pi\left(\mathcal{B}_{\mathbf{p}}^{(\infty)}\right) \cap \Theta=\emptyset$, and in particular $\mu_{\mathbf{p}}(\Theta)=0$, since $\mu_{\mathbf{p}}\left(\pi\left(\mathcal{B}_{\mathbf{p}}^{(\infty)}\right)\right)=\nu_{\mathbf{p}}\left(\mathcal{B}_{\mathbf{p}}^{(\infty)}\right)=1$ from Birkhoff's ergodic theorem.

Remark 3.1. Assume $M=\mathbb{N}$. Even though we are not concerned in this paper with self-similar sets generated by an infinite system of similitudes fulfilling strong OSC [11], it is worth mentioning that the proof of Theorem 2.1 remains valid in this case.

Remark 3.2. Let $\Sigma^{\infty}=\pi^{-1}\left(E \backslash \Theta^{*}\right)$. The mapping $\left.\pi\right|_{\Sigma^{\infty}}$ is a bijection onto $E \backslash \Theta^{*}$. This allows us to consider a unique fixed $\mathbf{i}_{x}=\pi^{-1}(x) \in \Sigma^{\infty}$ for each $x \in E \backslash \Theta^{*}$. We will use this fact often throughout this section. From the $\Theta$-lemma, $\mu_{\mathbf{p}}\left(E \backslash \Theta^{*}\right)=1$ for every positive probability $\mathbf{p}$ on $M$. More precisely, the $\Theta$-lemma implies that, for each $\mathbf{p} \in \mathcal{P}^{+}$, the measure spaces $\left(M^{\infty}, \mathcal{C}, \nu_{\mathbf{p}}\right)$ and $\left(E, \mathcal{C}_{\pi}, \mu_{\mathbf{p}}\right)$ are isomorphic, where $\mathcal{C}_{\pi}$ denotes the $\sigma$-algebra on $E$ induced by $\pi$ from the $\sigma$-algebra $\mathcal{C}$ generated by the class of cylinders on $M^{\infty}$.

From now on, let $\mathbf{p}$ be any fixed probability in $\mathcal{P}^{+}$. For convenience, we drop the index $\mathbf{p}$ from $\mu_{\mathbf{p}}$. We need some previous results in order to prove Theorems 2.2 and 2.4 .

Given a product measure $\mu \in \mathcal{M}^{+}$and a dimension function $\phi \in \mathcal{F}$, we define the following $\phi$-upper density of $\mu$ over geometrical cylinders at $x \in E$ :

$$
\bar{d}_{\mu}^{\phi}(x)=\sup \left\{\limsup _{k \rightarrow \infty} \frac{\mu\left(F_{\mathbf{i}(k)}\right)}{\phi\left(r_{\mathbf{i}(k)}\right)}: \mathbf{i} \in \pi^{-1}(x)\right\} .
$$

We will write $\bar{d}_{\mu}^{t}$ when $\phi(\xi)=\xi^{t}$.

For each ball $B$ of diameter $r>0$, consider the collection of sets $G(B)$ given by

$$
G(B)=\left\{F_{\mathbf{i}(k)}: \mathbf{i} \in M^{\infty}, F_{\mathbf{i}(k)} \cap B \neq \emptyset, r_{\mathbf{i}(k)} \leq r \text { and } r_{\mathbf{i}(k-1)}>r\right\} .
$$

The following lemma is essentially proved in [13].

Lemma 3.3. Let $B$ be any open ball with diameter $r>0$. Then

$$
\begin{gathered}
\text { card } G(B) \leq q<+\infty \text { independently of } r \\
\sum_{P \in G(B)} \phi(|P|) \leq q \phi(r) \text { for any } \phi \in \mathcal{F}
\end{gathered}
$$

We say that a family of sets $\left\{U_{i}\right\}_{i} \subset E$ is $\mu$-disjointed if $\mu\left(U_{i} \cap U_{j}\right)=0$ for $i \neq j$. From the $\Theta$-lemma, every covering of a subset of $E$ by geometric cylinders can be taken to be $\mu$-disjointed.

Lemma 3.4. Let $\mathcal{U}=\left\{C_{i}\right\}_{i}$ be a family of cylinders in $E$, i.e. $\mathcal{U} \subset\left\{E_{\mathbf{i}}: \mathbf{i} \in M^{*}\right\}$, such that $C_{i} \not \subset C_{j}$ for $i \neq j$. Then $\mathcal{U}$ is $\mu$-disjointed. 
Theorem 3.5. Let $A \subset E$ and let $a$ and $b$ be two positive constants. Then

(i) if $\sup _{x \in A} \bar{d}_{\mu}^{\phi}(x)<a$ and $A$ is $\mu$-measurable, then $H^{\phi}(A) \geq(a q)^{-1} \mu(A)$, where $q$ is as in $(3.7)$;

(ii) if $\inf _{x \in A} \bar{d}_{\mu}^{\phi}(x)>b$, then $H^{\phi}(A) \leq b^{-1} \phi^{*}$, where $\phi^{*}$ is given in (3.2).

Proof. (i) Let $n \in \mathbb{N}$, and consider the set

$$
\begin{aligned}
& A_{n}=\left\{\pi(\mathbf{i}) \in A \backslash \Theta^{*}: \mathbf{i} \in M^{\infty}, \text { for all } k \geq n\right. \\
& \left.\qquad \mu\left(F_{\mathbf{i}(k)}\right)<(a-\varepsilon) \phi\left(r_{\mathbf{i}(k)}\right) \text { for some } \varepsilon>0\right\} .
\end{aligned}
$$

Then $\left\{A_{n}\right\}_{n}$ is a non-decreasing sequence of sets with $\bigcup_{n} A_{n} \subseteq A$ and such that $\mu\left(A \backslash \bigcup_{n} A_{n}\right)=0$, because of the $\Theta$-lemma. Consider a $\delta$-covering $\mathcal{R}_{n}=\left\{B_{j}\right\}_{j}$ of $A_{n}$ by balls, with $u^{n}>\delta$, and take, for each $B_{j} \in \mathcal{R}_{n}$, the collection

$$
G_{j}(n)=\left\{P \in G\left(B_{j}\right): P \cap A_{n} \neq \emptyset\right\},
$$

where $G\left(B_{j}\right)$ is defined in (3.6). Now, for the chosen covering, we obtain

$$
\sum_{B_{j} \in \mathcal{R}_{n}} \phi\left(\left|B_{j}\right|\right) \geq q^{-1} \sum_{j} \sum_{P \in G_{j}(n)} \phi(|P|) \geq q^{-1} a^{-1} \sum_{j} \sum_{G_{j}(n)} \mu(P) \geq(q a)^{-1} \mu\left(A_{n}\right),
$$

where (3.8), the definition of $G_{j}(n)$, and the fact that $\bigcup_{j} \bigcup_{G_{j}(n)} P \supset A_{n}$ have been applied in that order. We thus obtain that $H_{\delta}^{\phi}(A) \geq H_{\delta}^{\phi}\left(A_{n}\right) \geq(a q)^{-1} \mu\left(A_{n}\right)$ for each $n$. Letting $\delta$ tend to 0 , we obtain $H^{\phi}(A) \geq(a q)^{-1} \mu(A)$, since $\mu\left(A_{n}\right)$ increases to $\mu(A)$.

(ii) Let $\varepsilon>0$ with $b-\varepsilon>0$. For each $x \in A$, choose some $\mathbf{i}_{x} \in \pi^{-1}(x)$ such that the inequality $\mu\left(F_{\mathbf{i}_{x}(k)}\right)>(b-\varepsilon) \phi\left(r_{\mathbf{i}_{x}(k)}\right)$ holds for infinitely many $k$. Now, for $\delta>0$, take $K \in \mathbb{N}$ such that $U^{K}<\delta$. For each $x \in A$ consider the integer

$$
k(x)=\min \left\{k: k>K, \mu\left(F_{\mathbf{i}_{x}(k)}\right)>(b-\varepsilon) \phi\left(r_{\mathbf{i}_{x}(k)}\right)\right\} .
$$

The collection $\left\{F_{\mathbf{i}_{x}(k)}: k=k(x), x \in A\right\}$ can be taken to be a $\mu$-disjointed $\delta$ covering, call it $\mathcal{U}$, of $A$ by cylinders. Therefore

$$
\sum_{\mathcal{U}} \phi\left(r_{\mathbf{i}(k)}\right) \leq(b-\varepsilon)^{-1} \sum_{\mathcal{U}} \mu\left(F_{\mathbf{i}(k)}\right) \leq(b-\varepsilon)^{-1} \mu(E)
$$

so that $\mathbf{H}_{\delta}^{\phi}(A) \leq(b-\varepsilon)^{-1}$ for an arbitrarily small $\varepsilon>0$, and the result follows from (3.3).

Lemma 3.6. Let $c=c(\mathbf{p})$, as given in $(2.4)$, and $f_{c}(\xi)=\left(2 \log \xi^{c} \log \log \log \xi^{c}\right)^{1 / 2}$. Then, for every $x \in B_{\mathbf{p}}$, there exists an $\mathbf{i} \in \pi^{-1}(x)$ such that

$$
\lim _{k \rightarrow \infty} \frac{f_{c}\left(r_{\mathbf{i}(k)}\right)}{(2 k \log \log k)^{1 / 2}}=1 .
$$

Proof. Let $R$ be the random variable on $M$ defined by $R(i)=\log r_{i}, i \in M$. Consider the Bernoulli process $\left\{R_{j}\right\}_{j}$, defined by $R_{j}=R \circ \operatorname{pr}_{1} \circ \tau^{j-1}$, where $\operatorname{pr}_{1}$ is the projection onto the first coordinate (see (2.11)) and $\tau$ is the shift mapping in (2.9). Observe that $S_{k}^{R}(\mathbf{i})=\log r_{\mathbf{i}(k)}$, where the notation in (2.10) is used.

Let $\mathbf{i} \in \pi^{-1}(x)$ be such that $\delta_{j}(\mathbf{i})=p_{j}$, for all $j \in M$. By the Strong Law of Large Numbers, it can be seen that $\lim _{k \rightarrow \infty} k^{-1} S_{k}^{R}(\mathbf{i})=c^{-1}$. Let $0<\varepsilon<-c^{-1}$, and take $k_{1}$ such that $k(1+c \varepsilon)<c \log r_{\mathbf{i}(k)}<k(1-c \varepsilon)$ holds for $k>k_{1}$. Let $k_{2}$ be large enough so that both

$$
\log \log (1+c \varepsilon) k / \log \log k>1+c \varepsilon
$$


and

$$
\log \log (1-c \varepsilon) k / \log \log k<1-c \varepsilon
$$

hold for $k>k_{2}$. Now, for $k>\max \left\{k_{1}, k_{2}\right\}$, the inequalities

$$
1+c \varepsilon<\frac{f_{c}\left(r_{\mathbf{i}(k)}\right)}{(2 k \log \log k)^{1 / 2}}<1-c \varepsilon
$$

hold, and thus (3.9) follows.

Given a Borel measure $\mu$, Rogers and Taylor [17] characterized those $\phi$-Hausdorff measures with respect to which $\mu$ is singular and those with respect to which $\mu$ is absolutely continuous in terms of the standard $\phi$-upper density (e.g. over dyadic intervals) of $\mu$. The key result needed to obtain such a characterization in terms of the density (3.5) defined over cylinders is the density theorem 3.5. It allows us to use the above mentioned characterization by means of the following version of the theorem in [17].

Rogers-Taylor Theorem. Let $\mu$ be a measure in $\mathbb{R}^{N}$ with $\sigma$-algebra $\mathcal{A}$, and let $\phi \in \mathcal{F}$. Assume there exist an $\mathcal{A}$-measurable function $d=d_{(\mu, \phi)}, d: \mathbb{R}^{N} \rightarrow[0,+\infty]$ and a constant $C>0$ such that

(i) if $d(x)<a$ for all $x \in A$ and $A \in \mathcal{A}$, then $a C H^{\phi}(A) \geq \mu(A)$;

(ii) if $d(x)>b$ for all $x \in A$, then $H^{\phi}(A) \leq C b^{-1}$.

Then

(a) $\mu$ is absolutely continuous w.r.t. $H^{\phi}$ if and only if $d(x)<+\infty \mu$-a.e.;

(b) $\mu$ is singular w.r.t. $H^{\phi}$ if and only if $d(x)=+\infty \mu$-a.e.;

(c) if $\mu \in \mathcal{M}^{+}$and $d=\bar{d}_{\mu}^{\phi}$ is the function defined in (3.5), then $\mu$ has an integral representation w.r.t. $H^{\phi}$ if and only if $0<d(x)<+\infty \mu$-a.e.

A Borel measure $\mu$ has an integral representation w.r.t. the measure $H^{\phi}$ if there exists a Borel set $E_{0}$ with $\phi$-Hausdorff measure $\sigma$-finite and an $H^{\phi}$-integrable function $f$ such that $\mu(A)=\int_{A \cap E_{0}} f(x) d H^{\phi}$ for all $A$ in the class of Borel sets.

Proof of Theorem 2.2. We take the upper density $\bar{d}_{\mu}^{\phi}(\cdot)$ defined in (3.5) as the function $d(\cdot)$ in the Rogers-Taylor Theorem. Some calculus allows us to check that $\phi_{\alpha}$ actually belongs to the class $\mathcal{F}$ defined in (3.1), and it satisfies (3.3). Notice that the measure-spaces isomorphism in Remark 3.2 along with Theorem 3.5 supply the hypothesis in the Rogers-Taylor Theorem for the self-similar measure $\mu$ and the point function $\bar{d}_{\mu}^{\phi}$.

Let $X$ be the random variable defined in (2.12). Recall that $\mathcal{E}[X]=0$ and $\mathcal{E}\left[X^{2}\right]=d(\mathbf{p})^{2}$. Consider the Bernoulli process $\left\{X_{k}\right\}_{k}$, where $X_{k}=X \circ \operatorname{pr}_{1} \circ \tau^{k-1}$ ( $\operatorname{pr}_{1}$ and $\tau$ were defined in (2.11) and (2.9) respectively). Let $\mathcal{G}$ be the family defined in (2.3). Note that the function $\phi_{\alpha} \in \mathcal{G}$ can be written as $\phi_{\alpha}(\xi)=$ $\xi^{s(\mathbf{p})} \exp \left(\alpha f_{c(\mathbf{p})}(\xi)\right)$, where $f_{c(\mathbf{p})}$ is as in Lemma 3.6. For $\alpha>0$, the $\phi_{\alpha}$-upper density at each $x \in E \backslash \Theta^{*}$ can be written as

$$
\bar{d}_{\mu}^{\phi_{\alpha}}(x)=\exp \left\{\limsup _{n \rightarrow \infty} f_{c}\left(r_{\mathbf{i}(n)}\right)\left(\frac{S_{n}^{X}(\mathbf{i})}{f_{c}\left(r_{\mathbf{i}(n)}\right)}-\alpha\right)\right\},
$$

where $\mathbf{i}=\pi^{-1}(x)$. From the $\Theta$-lemma, Lemma 3.6 and the Law of the Iterated Logarithm applied to the sequence of random variables $S_{n}^{X}$, we get from (3.10) that, for a set of full $\mu$-measure, $\bar{d}_{\mu}^{\phi_{\alpha}}(x)=+\infty$ if $0 \leq \alpha<d(\mathbf{p})$, and $\bar{d}_{\mu}^{\phi_{\alpha}}(x)=0$ if 
$\alpha>d(\mathbf{p})$. Therefore, from parts (a) and (b) in the Rogers-Taylor Theorem, parts (i) and (ii) of Theorem 2.2 follow.

Consider now, for $t>0$, the random variable $X^{(t)}$ defined by

$$
X^{(t)}(i)=\log p_{i}-t \log r_{i}, i \in M,
$$

and observe that the $t$-upper density at $x \in E \backslash \Theta^{*}$ can be written as

$$
\bar{d}_{\mu}^{t}(x)=\exp \left\{\limsup _{n \rightarrow \infty}\left(S_{n}^{X^{(t)}}\left(\pi^{-1}(x)\right)\right)\right\}
$$

so that the boundedness of the density $0<\bar{d}_{\mu}^{t}(x)<+\infty$ for $\mu$-almost every $x$ and for some $t>0$ is equivalent to the fact that

$$
-\infty<\limsup _{n \rightarrow \infty} S_{n}^{X^{(t)}}(\mathbf{i})<+\infty
$$

for $\nu$-almost every i. This boundedness requires $\mathcal{E}\left[X^{(t)}\right]=0$ and $\mathcal{E}\left[X^{(t)^{2}}\right]=0$, and thus $t=s(\mathbf{p})$ and $p_{i}=r_{i}^{s(\mathbf{p})}$ for $i \in M$. This is only plausible if $s(\mathbf{p})=s$. Part (c) in the Rogers-Taylor Theorem proves part (iii) of Theorem 2.2.

We now proceed to prove Theorem 2.4. We first remark on some general facts about Besicovitch sets. Observe that a normal Besicovitch set can also be written as

$$
B_{\mathbf{p}}=\bigcap_{n=1}^{\infty} \bigcup_{N=1}^{\infty} \bigcap_{k=N}^{\infty} \bigcap_{j \in M}\left\{\pi(\mathbf{i}): \mathbf{i} \in M^{\infty} \text { and } p_{j}-\frac{1}{n}<\delta_{j}(\mathbf{i}, k)<p_{j}+\frac{1}{n}\right\},
$$

where the notation $\delta_{j}(\mathbf{i}, k)$ stands for the frequency of the symbol $j$ in the finite sequence $\mathbf{i}(k)$. The bracketed set in that expression is a finite union of cylinders $E_{\mathbf{j}}$, $\mathbf{j} \in M^{*}$, so that it is a closed set. This shows that a normal Besicovitch set is an $F_{\sigma \delta}$ set, and as a consequence it is a Borel set. Moreover, the normal Besicovitch sets are non-compact self-similar $\left(S \Psi\right.$-invariant) sets, i.e. $S \Psi\left(B_{\mathbf{p}}\right)=B_{\mathbf{p}}$ for any $\mathbf{p}$ in $M$. Observe also that $B_{\mathbf{p}}$ is dense in $E$ for any $\mathbf{p} \in \mathcal{P}^{+}$. Notice that the same properties hold for the supernormal Besicovitch sets $B_{\mathbf{p}}^{(\infty)}$ and for the set $L_{\mathbf{p}}$.

Proof of Theorem 2.4, part (i). Given (2.1) and the definitions (2.6) and (2.8), we only need to prove that $\operatorname{dim} B_{\mathbf{p}} \leq s(\mathbf{p})$. This can be directly obtained from Theorem 3.5. Let $t>s(\mathbf{p})$, and consider the random variable $X^{(t)}$ defined in (3.11). Notice that the $t$-upper density at any $x \in B_{\mathbf{p}}$ satisfies

$$
\bar{d}_{\mu}^{t}(x) \geq \exp \left\{\limsup _{n \rightarrow \infty} S_{n}^{X^{(t)}}(\mathbf{i})\right\}
$$

for any $\mathbf{i} \in \pi^{-1}(x)$. Since $\mathcal{E}\left[X^{(t)}\right]>0$, and

$$
\lim _{n \rightarrow \infty} \frac{1}{n} S_{n}^{X^{(t)}}\left(\mathbf{i}_{x}\right)=\mathcal{E}\left[X^{(t)}\right]
$$

for some $\mathbf{i}_{x} \in \pi^{-1}(x)$, we get from (3.12) that $\bar{d}_{\mu}^{t}(x)=+\infty$ for all $x \in B_{\mathbf{p}}$. From Theorem 3.5 it follows that $H^{t}\left(B_{\mathbf{p}}\right)=0$, for $B_{\mathbf{p}}$ is a full $\mu$-measure set. Since $t>s(\mathbf{p})$ is arbitrary, we have the desired inequality.

Proof of Theorem 2.4, part (ii). Let $\mu_{s}$ denote the self-similar measure associated with $\mathbf{p}_{s}$. By the uniqueness of the invariant measure associated with the pair $(\Psi, \mathbf{p})$ (see [10]), the measures $\mu_{s}$ and $H^{s}$ coincide up to a constant factor. Since $\mu_{s}\left(E \backslash B_{\mathbf{p}_{s}}\right)=0$, then $H^{s}\left(E \backslash B_{\mathbf{p}_{s}}\right)=0$, and therefore $H^{s}(E)=H^{s}\left(B_{\mathbf{p}_{s}}\right)$. This 
measure is known to be finite and positive [10]. From part (iv) of Theorem 2.4 (see below) we know that $\mu_{\mathbf{p}}\left(L_{\mathbf{p}}\right)=1$ for every $\mathbf{p} \in \mathcal{P}^{+}$. This proves (ii) in Theorem 2.4 , for the reasoning above also applies to the sets $B_{\mathbf{p}_{s}}^{(\infty)}$ and $L_{\mathbf{p}_{s}}$.

Since Besicovitch sets are invariant under the set mapping $S \Psi$, part (iii) of Theorem 2.4 follows from the more general result below.

Proposition 3.7. Let $0<t<s$ and $\phi(x)=x^{t} g(x)$, with $g$ non-increasing in some interval $\left(0, \delta_{0}\right)$. Then, either $H^{\phi}(B)=0$ or $H^{\phi}(B)=+\infty$, for any $S \Psi$-invariant set $B$ such that $\operatorname{dim} B>\operatorname{dim}(\Theta \cap B)$.

Proof. We first prove that $H^{\phi}(\varphi(A)) \geq r^{t} H^{\phi}(A)$ for any similitude $\varphi$ with contraction ratio $r \leq 1$, and for any $A \subset E$. Let $0<\delta<r \delta_{0}$ and consider a $\delta$-covering $\left\{V_{i}\right\}_{i}$ of $\varphi(A)$. If we write $U_{i}=\varphi^{-1}\left(V_{i}\right)$ for every $i$, we have

$$
\sum_{i} \phi\left(\left|V_{i}\right|\right)=r^{t} \sum_{i}\left|U_{i}\right|^{t} g\left(r\left|U_{i}\right|\right) \geq r^{t} \sum_{i}\left|U_{i}\right|^{t} g\left(\left|U_{i}\right|\right)=r^{t} \sum_{i} \phi\left(\left|U_{i}\right|\right) .
$$

Since $\left\{U_{i}\right\}_{i}$ is a $\delta / r$-covering of $A$, this shows that $H_{\delta}^{\phi}(\varphi(A)) \geq r^{t} H_{\delta / r}^{\phi}(A)$, and therefore the inequality $H^{\phi}(\varphi(A)) \geq r^{t} H^{\phi}(A)$ holds.

Assume that $0<H^{\phi}(B)<+\infty$ for an $S \Psi$-invariant set $B$ such that $\operatorname{dim} B>$ $\operatorname{dim} B \cap \Theta$. Then we have

$$
H^{\phi}(B)=H^{\phi}(S \Psi(B))=\sum_{i \in M} H^{\phi}\left(\varphi_{i}(B)\right) \geq \sum_{i \in M} r_{i}^{t} H^{\phi}(B)>H^{\phi}(B),
$$

since $t<s$ and $\sum_{i \in M} r_{i}^{x}$ is a decreasing function of $x$. This contradiction implies that $B$ has $H^{\phi}$-measure zero or infinity.

Observe that Proposition 3.7 holds for the gauge $\phi(\xi)=\xi^{t}, t \neq s$. This implies that there is no $S \Psi$-invariant $t$-set in $E$ for $\operatorname{dim} \Theta<t<s$. Notice also that Proposition 3.7 applies to every $S \Psi$-invariant subset of non-overlapping self-similar sets, and to all supernormal Besicovitch sets since the intersection $B_{\mathbf{p}}^{(\infty)} \cap \Theta$ is void (see the proof of the $\Theta$-lemma). The result still holds for normal Besicovitch sets $B_{\mathbf{p}}$ such that $\operatorname{dim}\left(B_{\mathbf{p}} \cap \Theta\right)<\operatorname{dim} B_{\mathbf{p}}=s(\mathbf{p})$.

Remark 3.8. Assume the system $\Psi$ is disjointed. The multifractal component of level $\alpha$ of the measure $\mu_{\mathbf{p}}$ can be defined by

$$
K(\alpha)=\left\{\pi(\mathbf{i}): \lim _{k \rightarrow \infty} \frac{\log \mu_{\mathbf{p}}\left(F_{\mathbf{i}(k)}\right)}{\log r_{\mathbf{i}(k)}}=\alpha\right\}
$$

(see [5, Theorem 3.3]). Cawley and Mauldin asked in [5, Question 2.18] whether $0<H^{f(\alpha)}(K(\alpha))<+\infty$, where $f(\alpha)=\operatorname{dim} K(\alpha)$. More generally, they asked whether there exists a function $L(\xi)$ such that $\lim _{\xi \rightarrow 0^{+}} \log L(\xi) / \log \xi=0$ and $0<H^{h}(K(\alpha))<+\infty$, where $h(\xi)=\xi^{f(\alpha)} L(\xi)$. Since the sets $K(\alpha)$ are $S \Psi$ invariant, Proposition 3.7 shows that the $h$-Hausdorff measure of $K(\alpha)$ is either zero or infinity for any $h$ as above with $L$ non-increasing. In particular, the sets $K(\alpha)$ are not $f(\alpha)$-sets.

Proof of Theorem 2.4, part (iv). From part (i) in Theorem 2.4 and the definition of $L_{\mathbf{p}}$, it follows that $\operatorname{dim} L_{\mathbf{p}} \leq s(\mathbf{p})$. 
We proceed as in the proof of Theorem 2.2. Let $X$ be the random variable in (2.12). For every $x \in L_{\mathbf{p}}$, the $\phi_{\alpha}$-upper density can be written as

$$
\bar{d}_{\mu}^{\phi_{\alpha}}(x)=\exp \left\{\limsup _{n \rightarrow \infty} f_{c}\left(r_{\mathbf{i}_{x}(n)}\right)\left(\frac{S_{n}^{X}\left(\mathbf{i}_{x}\right)}{f_{c}\left(r_{\mathbf{i}_{x}(n)}\right)}-\alpha\right)\right\} .
$$

Since $d(\mathbf{p})>0$ forp $\neq \mathbf{p}_{s}$, it followsfrom (2.13), Lemma 3.6, and (3.13) that $\bar{d}_{\mu}^{\phi_{\alpha}}(x)=+\infty$ for all $0 \leq \alpha<d(\mathbf{p})$. The Law of the Iterated Logarithm implies that the set $L_{\mathbf{p}}$ carries the whole measure $\mu_{\mathbf{p}}$, for $B_{\mathbf{p}}^{(\infty)}$ also does. Therefore, Theorem 3.5 gives that $H^{\phi_{\alpha}}\left(L_{\mathbf{p}}\right)=0$ for $0 \leq \alpha<d(\mathbf{p})$. This gives $H^{s(\mathbf{p})}\left(L_{\mathbf{p}}\right)=0$, because $H^{s(\mathbf{p})} \leq H^{\phi_{\alpha}}$ for all $\alpha>0$.

Closing Remark. Some natural extensions of the research presented in this paper are to generalize to the case $M=\mathbb{N}$ the results obtained here, and to solve the same questions for the packing dimension [20]. Some results concerning these issues are obtained in [12].

\section{REFERENCES}

[1] C. Bandt, Deterministic fractals and fractal measures, in Lecture notes of the School on Measure Theory and Real Analysis, Grado, Italy, 1991, Rend. Ist. Mat. Univ. Trieste 23 (1991), 1-40 (1993). MR 95e:28002

[2] M. Barnsley, Fractals everywhere, Academic Press, 1988. MR 90e:58080

[3] A.S. Besicovitch, On the sum of digits of real numbers represented in the dyadic system, Math. Ann. 110 (1934), 321-330.

[4] P. Billingsley, Hausdorff dimension in probability theory, Illinois J. Math. 4 (1960), 187-209. MR 24:A 1750

[5] R. Cawley and R.D. Mauldin, Multifractal decomposition of Moran fractals, Adv. Math. 92 (1992), 196-236. MR 93b:58085

[6] M.J.P. Cooper, The Hausdorff measure of the Besicovitch-Eggleston set, preprint.

[7] A. Deliu, J.S. Geronimo, R. Shonkwiler and D. Hardin, Dimensions associated with recurrent self-similar sets, Math. Proc. Cambridge Phil. Soc. 110 (1991), 327-336. MR 92g:58069

[8] H.G. Eggleston, The fractional dimension of a set defined by decimal properties, Quart. J. Math. Oxford Ser. 20 (1949), 31-36. MR 11:88e

[9] K.J. Falconer, The Multifractal spectrum of statistically self-similar measures, J. Theoret. Probab. 7 (1994), 681-702. MR 95m:60076

[10] J.E. Hutchinson, Fractals and self-similarity, Indiana Univ. Math. J. 30 (1981), 713-747. MR 82h: 49026

[11] M. Morán, Hausdorff measure of infinitely generated self-similar sets, Monatsh. Math. 122 (1996), 387-399. MR 97j:28015

[12] M. Morán and J.-M. Rey, Geometry of self-similar measures, Ann. Acad. Sci. Fenn. Mathematica 22 (1997), 365-386.

[13] P.A.P. Moran, Additive functions of intervals and Hausdorff measure, Proc. Cambridge Phil. Soc. 42 (1946), 15-23. MR 7:278f

[14] N. Patzschke, Self-conformal multifractal measures, preprint.

[15] F. Przytycki, M. Urbański and A. Zdunik, Harmonic, Gibbs and Hausdorff measures on repellers for holomorphic maps, I, Ann. of Math. 130 (1989), 1-40. MR 91i:58115

[16] M.S. Raghunathan, A proof of Oseledec's multiplicative ergodic theorem, Israel J. Math. 32 (1978) 356-362. MR 81f:60016

[17] C.A. Rogers and S.J. Taylor, Functions continuous and singular with respect to a Hausdorff measure, Mathematika 8 (1961), 1-31. MR 24:A200

[18] A. Schief, Separation properties for self-similar sets, Proc. Amer. Math. Soc. 122 (1994), 111-115. MR 94k:28012

[19] M. Smorodinsky, Singular measures and Hausdorff measures, Israel J. Math. 7 (1969), 203206. MR 40:3589

[20] C. Tricot, Two definitions of fractional dimension, Math. Proc. Cambridge Phil. Soc. 91 (1982), 57-74. MR 84d:28013 
[21] P. Walters, An introduction to ergodic theory, Springer-Verlag, 1982. MR 84e:28017

[22] L.S. Young, Dimension, entropy and Liapunov exponents, Ergodic Theory Dynam. Systems 2 (1982), 109-124. MR 84h:58087

Departamento de Análisis Económico, Universidad Complutense, Campus de SomoSAguas, 28223 Madrid. Spain

E-mail address: ececo06@sis.ucm.es

E-mail address: ececo07@sis.ucm.es 